\title{
Antibiotic resistance among Salmonella enterica serovars Typhi and Paratyphi A in Pakistan (2001-2006)
}

\author{
Rumina Hasan ${ }^{1}$, Afia Zafar ${ }^{1}$, Zohair Abbas ${ }^{2}$, Vikram Mahraj ${ }^{1}$, Faisal Malik ${ }^{1}$, Anita Zaidi \\ ${ }^{1}$ Departments of Pathology \& Microbiology and ${ }^{2}$ Pediatrics, Aga Khan University Hospitals, Karachi, Pakistan
}

\begin{abstract}
Objectives: To compare antimicrobial resistance in S. Typhi and S. Paratyphi A isolates from Pakistan.

Methods: Blood samples were collected through > 175 laboratory collection points in major cities and towns across the country. The study included 3,671 S. Typhi and 1,475 S. Paratyphi A isolates (2001-2006). Multidrug resistance (MDR) was defined as resistance to first-line agents co-trimoxazole, chloramphenicol and ampicillin.

Results: In total, $79.3 \%$ S. Typhi and $59.9 \%$ S. Paratyphi A were isolated from patients under 15 years of age. During the study period, the MDR rate increased in S. Typhi $(34.2$ to $48.5 \% \mathrm{p}<0.001)$, but decreased in S. Paratyphi A $(44.5$ to $8.6 \% \mathrm{p}<0.001)$. Quinolone resistance $(\mathrm{MIC}>1 \mu \mathrm{g} / \mathrm{ml})$ increased in both $S$. Typhi $(1.6$ to $64.1 \% \mathrm{p}<0.001)$ and $S$. Paratyphi $A(0$ to $47 \% \mathrm{p}<0.001)$. The increase in the proportion of strains showing high level quinolone resistance (MIC $>4 \mu \mathrm{g} / \mathrm{ml}$ ) was greater in $\mathrm{S}$. Paratyphi $\mathrm{A}$ when compared to S. Typhi. Resistance to first-line drugs was higher in those $<15$ years of age whereas quinolone resistance was higher in older patients.

Conclusion: Differences between S. Typhi and S. Paratyphi A, in terms of evolution of resistance to first-line agents and to quinolones, are evident in this population. The rapid increase in quinolone resistance in S. Paratyphi $A$ when compared to $S$. Typhi is concerning and requires further study.
\end{abstract}

Key Words: Salmonella, Antibiotic resistance, Typhi, Paratyphi, Pakistan

J Infect Developing Countries 2008; 2(4): 289-294.

Received 19 June 2008 - Accepted 22 July 2008

Copyright (C) 2008 Hasan et al. This is an open access article distributed under the Creative Commons Attribution License, which permits unrestricted use, distribution, and reproduction in any medium, provided the original work is properly cited.

\section{Introduction}

Enteric fever is an acute, life-threatening, febrile illness caused by Salmonella enterica serovars Typhi, Paratyphi A, B and C, and is estimated to be responsible for 21 million illnesses and 200,000 deaths worldwide annually. It is further associated with significant economic consequences in developing countries [1]. The incidence rate of enteric fever in central and south Asia is estimated at more than 100 cases/100,000 population per year with the highest burden of disease seen in children [1-5].

Emergence of antimicrobial resistance, in particular multidrug resistance (MDR, resistance to chloramphenicol, ampicillin and co-trimoxazole) has greatly complicated disease management $[6,7]$. MDR S. Typhi first reported from Bangladesh in 1986 and from Pakistan in 1988 is currently endemic in South Asia [8-10]. Resistance in S. Paratyphi $A$ is also emerging with a number of reports documenting prevalence of MDR $S$. Paratyphi A in this region [11-12].

Fluoroquinolones are regarded as the treatment of choice for enteric fever in adults. However, limited access to reliable clinical microbiology facilities and the ready availability and excessive use of low-cost substandard generics of fluoroquinolone has led to the emergence of fluoroquinolone resistance [13]. Clinical failures and inadequate responses to therapy are documented in patients infected with Salmonella strains with reduced susceptibility to fluoroquinolones (MIC > $0.125 \mathrm{ug} / \mathrm{ml}$ ) [14-17]. Point mutations in chromosomal genes (gyrA, gyrB, parC, and parE) resulting in strains with reduced susceptibility as well as plasmid-encoded transferable resistance to fluoroquinolones have been reported from many countries [18-24].

In Pakistan, the incidence of culture-proven enteric fever in children is estimated at 170 per 100,000 of the population, whereas serology 
based incidence is estimated at 710 per 100,000 of the population [25]. Published reports suggest that the MDR rate amongst $S$. Typhi in the country decreased from $50 \%$ in 1995 to $20 \%$ in 2001 [6] while the rate in $S$. Paratyphi $A$ increased from $14 \%$ in 1996 to $44 \%$ in 2003 [26]. The aim of this study was to explore the current level of resistance to first-line agents and to quinolones amongst $S$. enterica serovars Typhi and Paratyphi A in Pakistan. We further aimed to study the change in antimicrobial resistance among these organisms over a six-year period, 2001-2006.

\section{Material and Methods}

S. enterica serovars Typhi and Paratyphi A isolated from blood cultures at the Aga Khan University's Clinical Microbiology Laboratory were included in the study. Aga Khan University Hospital is a 550-bed, tertiary care centre located in Karachi, Pakistan. Its clinical laboratory receives samples from both in-patients and outpatients. The latter are collected through more than 175 collection units located in all major cities and towns across the country.

From January 2001 to December 2006, a total of 175,987 blood specimens were submitted to the clinical laboratory. Blood specimens were processed in the BACTEC system (Becton and Dickenson, USA). Positive blood samples were further cultured on sheep blood, chocolate and McConkey agar plates and incubated at $35^{\circ}-36^{\circ} \mathrm{C}$ for 24 to 48 hours. Suggestive non-lactosefermenter colonies were identified on the basis of standard biochemical tests using API20E and agglutination with Salmonella 09, Vi-specific and $\mathrm{Hd}$ antisera (S. Typhi), and with 02 and $\mathrm{Ha}$ antisera ( $S$. Paratyphi A) (Difco laboratories). Antimicrobial susceptibility tests were performed using the Kirby Bauer disc diffusion method in accordance with the Clinical and Laboratory Standard Institute (CLSI) guidelines. Discs used included chloramphenicol $(30 \mu \mathrm{g})$, ampicillin $(10 \mu \mathrm{g})$, co-trimoxazole $(1.25 / 23.75 \mu \mathrm{g})$, nalidixic acid $(30 \mu \mathrm{g})$ and ofloxacin $(5 \mu \mathrm{g})$ [27]. Isolates resistant to chloramphenicol, ampicillin, and cotrimoxazole were termed multi-drug resistant (MDR).

Strains resistant to nalidixic acid were further confirmed as being fluoroquinolone resistant using minimum inhibitory concentration (MIC). MIC was determined by the standard agar dilution method using Muller Hinton agar as described in CLSI using Escherichia coli ATCC 25922 as a control 27. A breakpoint of $0.125 \mu \mathrm{g} / \mathrm{ml}$ was used as reduced susceptibility, and MIC of $>1.0 \mu \mathrm{g} / \mathrm{ml}$ as resistance to quinolones [28].

The data was extracted from the computerised hospital information system and transferred to SPSS 15.0 (SPSS Inc., Chicago, IL, USA) for statistical analysis. In descriptive analysis, means and standard deviations of the continuous variables and percentages of the categorical variables were computed. In inferential analysis, comparisons between resistant and sensitive strains by gender, age and location were conducted through chi square or Fisher's exact test where appropriate. Year-wise trends were assessed through a chi square test for trend. A pvalue of less than or equal to $5 \%$ was considered as statistically significant.

\section{Results}

A total of 3,671 Salmonella Typhi and 1,475 Salmonella Paratyphi A strains isolated between 2001 and 2006 were studied. In both S. Typhi and $S$. Paratyphi $A$, the majority of isolates were from children under the age of 15 years. However, as shown in Table 1, there was a greater representation of S. Typhi $(79.3 \%)$ in the under 15 group as compared to S. Paratyphi A (59.9\%) $p<0.001$. No gender related difference in isolation rates of $S$. Typhi and $S$. Paratyphi $A$ were detected.

Table 1. Descriptive characteristics of patients positive for S. enterica serovar Typhi and Paratyphi A (20012006).

\begin{tabular}{llll}
\hline Characteristics & $\begin{array}{l}\text { S. Typhi } \\
\mathbf{n}(\%)\end{array}$ & $\begin{array}{l}\text { S. para } \boldsymbol{A} \\
\mathbf{n}(\%)\end{array}$ & p value \\
\hline Age in years & & & \\
$\quad<15$ Years & $2910(79.3)$ & $895(59.9)$ & \\
15 \& above & $735(20.0)$ & $592(39.8)$ & $<0.001^{*}$ \\
$\quad$ Missing information & $26(0.7)$ & $8(0.5)$ & \\
\hline Gender & & & \\
$\quad$ Male & $2125(57.9)$ & $842(56.3)$ & \\
$\quad$ Female & $1544(42.0)$ & $651(43.5)$ & 0.316 \\
$\quad$ Missing information & $2(0.1)$ & $2(0.1)$ & \\
\hline Total Lumber & 3671 & 1495 & \\
\hline p-value calculated through chi squared test excluding the missing values, \\
significance $\left(^{*}\right)$ p $<0.05$.
\end{tabular}

During the 6-year study period, resistance to co-trimoxazole, ampicillin and chloramphenicol showed a steady increase in S. Typhi $(p<0.001)$. Conversely, the trend in S. Paratyphi $A$ saw the MDR rate fall from $44.5 \%$ in 2001 to $8.6 \%$ in 2006 
$(p<0.001)$. During the same period, an increase in fluoroquinolone resistance was noted in both the groups $(p<0.001)$ (Figures 1A \& 1B).

Figure 1. A (upper): Antimicrobial resistance; $S$. enterica serovar Typhi (2001-2006). B (lower): Antimicrobial resistance; S. enterica serovar Paratyphi A (2001-2006).

Resistance is expressed as a percentage of the total strains isolated per year. The number of strains tested per year is depicted; ( $n$ value). Resistance to individual first-line agents as well as MDR increased in S. Typhi $(p<0.001)$, but decreased in $S$. Paratyphi $(p<0.001)$.

Resistance to Ofloxacin increased significantly in both organisms studied $(p<0.001)$.
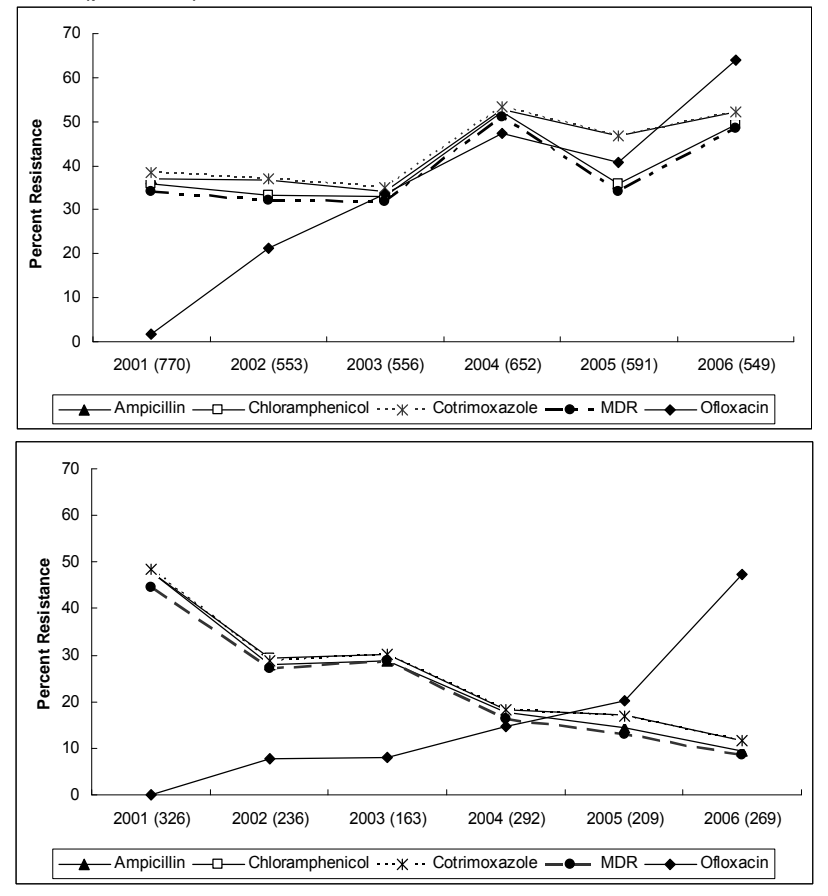

Comparison of the cumulative data (20012006) for S. Typhi and S. Paratyphi A suggests a significant association between MDR and ofloxacin resistance in S. Typhi; $59.8 \%$ of MDR S. Typhi isolates were resistant to ofloxacin compared to only $16.5 \%$ of sensitive $S$. Typhi isolates $(p<0.001)$. This trend, however, did not hold true for $S$. Paratyphi A where a negative association between MDR and quinolone resistance was apparent: $5.4 \%$ of MDR S. Paratyphi A isolates were resistant to ofloxacin as opposed to $19.6 \%$ of sensitive isolates $(p<0.001)$.

Analysis of MIC levels to ofloxacin in both the study organisms showed that the level of quinolone resistance in terms of MIC values was increasing in both $S$. Typhi and S. Paratyphi A over the 6-year study period. The increase in percentage of strains with ofloxacin MIC levels $(>4 \mathrm{mg} / \mathrm{ml})$ was, however, markedly greater in $S$.
Paratyphi A $0 \% \quad(2001)$ to $28 \%$ (2006) as compared to S. Typhi 0\% (2001 to 9\%(2006) (Figures 2A \& 2B).

Figure 2. A: MIC to Ofloxacin; S. enterica serovar Typhi; B: MIC to Ofloxacin; S. enterica serovar Paratyphi A.

Minimum Inhibitory Concentration (MIC) values for S. Typhi and paratyphi $A$ that were naladixic acid resistant on initial screening (2001-2006).

The number of strains tested per year is shown ( $\mathrm{n}$ value).

MIC values (range 0.125 to $>4.0 \mu \mathrm{g} / \mathrm{ml}$ ) are as follows as depicted in figure legend.
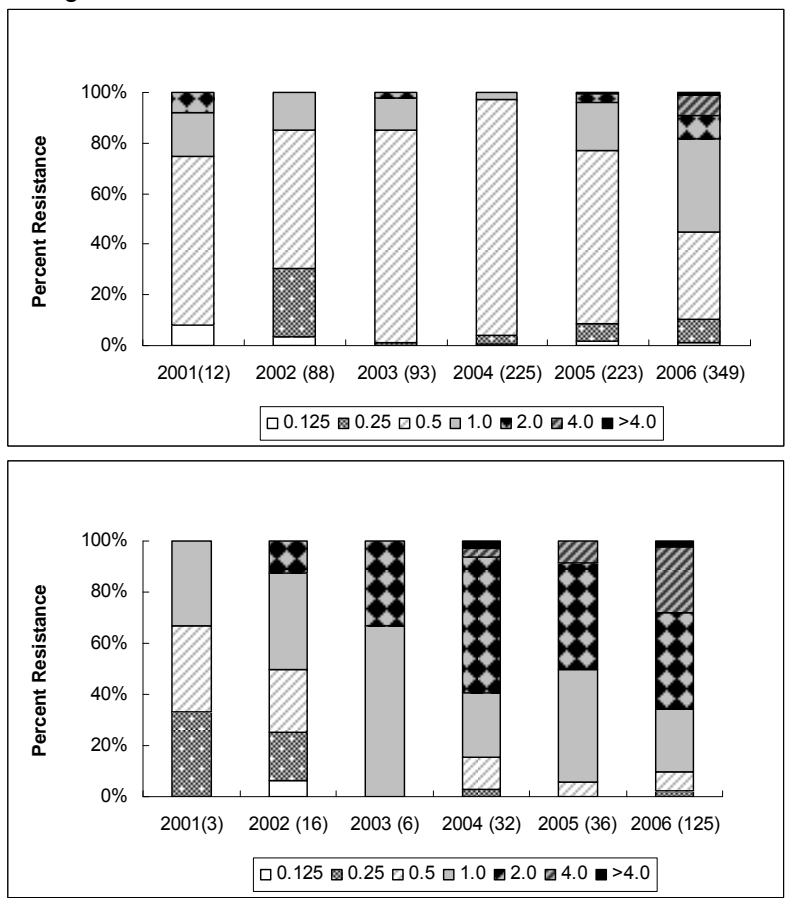

Isolates from younger patients (<15years) were more likely to be resistant to ampicillin $(p=0.017)$ and co-trimoxazole $(p=0.016)$ in the case of $S$. Typhi, and to ampicillin $(p=0.002)$, chloramphenicol $(p<0.001)$ and co-trimoxazole $(p<0.001)$ in cases of $S$. Paratyphi A. Resistance to fluoroquinolones in both organisms, however, was more likely in the older age groups $(p<0.001)$ (Table 2).

Table 2. Distribution of antibiotic sensitivity in $S$. enterica serovars Typhi and Paratyphi isolates according to age (2001-2006).

\begin{tabular}{|c|c|c|c|c|c|c|c|}
\hline \multirow{2}{*}{ Feature } & \multirow{2}{*}{$\begin{array}{c}\text { Age } \\
(\mathrm{y})\end{array}$} & \multirow{2}{*}{$\mathrm{n}$} & Amp & Chloram & Cotrim & MDR & Oflox \\
\hline & & & $\%-R$ & $\%-R$ & $\%-R$ & $\%-R$ & $\%-R$ \\
\hline \multirow{3}{*}{$\begin{array}{c}\mathrm{ST} \\
(\mathrm{n}: 3671)\end{array}$} & $<15$ & 2909 & 44.2 & 40.5 & 44.8 & 39.3 & 31.2 \\
\hline & $\geq 15$ & 735 & 39.4 & 38.2 & 39.9 & 36.1 & 41.4 \\
\hline & \multicolumn{2}{|c|}{$P$} & $0.017^{*}$ & 0.270 & $0.016^{*}$ & 0.107 & $<0.001^{*}$ \\
\hline
\end{tabular}




\begin{tabular}{crrlllll}
\hline \multirow{2}{*}{$\mathrm{SPT}$} & $<15$ & 895 & 27.9 & 29.5 & 29.9 & 26.6 & 13.5 \\
$(\mathrm{n}: 1495)$ & $\geq 15$ & 592 & 20.7 & 21.3 & 20.3 & 19.1 & 20.8 \\
\cline { 2 - 7 } & \multicolumn{2}{c}{$\mathrm{P}$} & $0.002^{*}$ & $<0.001^{*}$ & $<0.001^{*}$ & $0.001^{*}$ & $<0.001^{*}$
\end{tabular}

ST= S. Typhi; SPT = S. Paraty-phi A; Amp = Ampicillin; Chloram = Chloramphenicol; Cotrim = Co-trimoxzole; $\mathrm{MDR}=$ Multi drug resistant $=$ Resistant to Ampicillin, Chloramphenicol and Co-trimoxazole; Oflox = Ofloxacin. Age analysis was not conducted for $27 \mathrm{~S}$. Typhi and $8 \mathrm{~S}$. Paratyphi A patients due to missing information. p-value calculated through chi squared test excluding the missing values, significance $\left({ }^{*}\right) p<0.05$.

\section{Discussion}

We compared resistance trends of S. enterica serovars Typhi and S. Paratyphi A isolated between 2001 and 2006. During that period, the isolation rate of $\mathrm{S}$. Typhi was significantly greater than that of $S$. Paratyphi $A$. This finding is in agreement with published data indicating typhoid fever as more prevalent than paratyphoid in this region $[12,25,29]$.

A higher frequency of both Typhi and Paratyphi $A$ in the younger age group, reported in this study, also concurs with published literature showing a greater burden of enteric fever in children as compared to adults [2-5,25]. Comparison of strain distribution between adults and children showed that children bore $79.3 \%$ of the S. Typhi burden as compared to $59.9 \%$ of $S$. Paratyphi A. This difference, while similar to that reported from India $[29,30]$, is difficult to explain. It is possible to hypothesise that, when compared to $S$. Typhi, the lower overall prevalence of $S$. Paratyphi $A$ in the community reduces chances of exposure and hence likelihood of disease in the younger age group.

The increase in resistance amongst $S$. Typhi to the first-line agents co-trimoxazole, chloramphenicol and ampicillin is concerning and likely to reflect persistence and dissemination of resistant $S$. Typhi strains within our community. In contrast, the decrease in resistance to the first-line agents in $S$. Paratyphi $A$ is encouraging but surprising in view of earlier reports of upward resistance trends among $S$. Paratyphi $A$ in the country [26]. A similar decrease in resistance among $S$. Paratyphi A has also been documented in a recent report from India [31]. It is possible to link this downward trend in resistance among $S$. Paratyphi A to the fact that third-generation cephalosporins have, to a large extent, replaced co-trimoxazole, chloramphenicol and ampicillin as the treatment of choice for enteric fever in children. Our data further suggests, however, that the resistant strains of $S$. Paratyphi $A$ have not succeeded in establishing themselves in the community. It has been suggested that antimicrobial resistance in Salmonella may be associated with a fitness cost [32]. Whether the resistance in $S$. Paratyphi $A$ has an associated fitness cost needs to be investigated. S. Paratyphi $A$ has been shown to harbour resistance plasmids [33]. It is possible that in the absence of antibiotic pressure, these plasmids are not retained but may be re-acquired when antibiotic pressure is reimposed [34].

Fluoroquinolone resistance in S. Typhi as well as in S. Paratyphi A increased during the 6-year study period, both in terms of frequency and MIC levels. Fluoroquinolones available over the counter in the country are used to treat a number of infections including urinary and respiratory tract infections. Resistance is most likely a consequence of their widespread use in the community. Such increase in resistance to fluoroquinolones amongst $S$. enterica has been widely reported [14-17]. However, the sharp rise in S. Paratyphi A MIC levels as compared to $S$. Typhi is concerning. This observation, suggesting that $S$. Paratyphi $A$ is more prone to developing fluoroquinolone resistance, requires further exploration.

The difference in resistance between the younger and older age groups noted in this study is difficult to explain. Moreover, our finding is in contrast to data from Delhi reporting no difference in antimicrobial resistance across age groups in their patients [30]. Our data of higher resistance to co-trimoxazole, chloramphenicol and ampicillin among isolates from the younger age group and greater fluoroquinolone resistance in strains from older patients is consistent with antimicrobial usage in these two patient groups. The difference between our study and that from Delhi may well be related to the fact that, in the Delhi study, patients with history of antimicrobial therapy were excluded. The finding of age-related difference in antimicrobial resistance is also surprising given that risk factors for typhoid reportedly include a recent case in the family $[35,36]$. However, typhoid fever in endemic settings has been linked with high-dose exposure from multiple sources and therefore the possibility of an extra-familial source cannot be excluded in such cases [37]. Prior antimicrobial usage is reported to be a risk factor for typhoid $[37,38]$. However, the impact of such antimicrobials on the resistance pattern of the infecting organisms needs to be considered. The 
role of commensal flora within host intestinal flora harbouring resistance plasmids and contributing to development of resistance $S$. enterica serovars is also a distinct possibility requiring exploration.

In conclusion, our data suggests that there appear to be differences between $S$. enterica serovar Typhi and S. Paratyphi $A$ in terms of resistance trends to first-line agents, cotrimoxazole, chloramphenicol and ampicillin. These differences need to be explored further. Increasing resistance to fluoroquinolones is alarming and of particular concern is the rapid rise in MIC levels among S. Paratyphi A. Similarly, differences in resistance patterns between younger and older age groups need to be further studied.

\section{Acknowledgements}

This study was supported by a grant from the Joint PakistanUS Academic and Research Program HEC/MoST/USAID. We would also like to thank the faculty and staff of the Clinical Microbiology Laboratory of the Aga Khan University Hospital, Karachi, for their support and help in this study.

\section{References}

1. Crump J., Luby SP, Mintz ED (2004) The global burden of typhoid fever. Bull World Health Organ 82:346-353.

2. Sinha A, Sazawal S, Kumar R, Sood S, Reddaiah VP, Singh B et al. (1999) Typhoid fever in children aged less than 5 years. Lancet 354:734-7.

3. Brooks WA, Hossain A, Goswami D, Nahar K, Alam K, Ahmed N, et al. (2005) Bacteremic typhoid fever in children in an urban slum, Bangladesh. Emerg Infect Dis 11:326-9.

4. Siddiqui FJ, Rabbani F, Hasan R, Nizami SQ, Bhutta ZA (2006) Typhoid fever in children: some epidemiological considerations. Int J Infect Dis 10:215-22. Epub 2006 Jan 23.

5. Grahm SM (2002) Salmonellosis in children in developing and developed countries and populations. Curr Opin Infec Dis 15:507-12.

6. Okeke IN, Laxminarayan R, Bhutta ZA, Duse AG, Jenkins $\mathrm{P}$, O'Brien TF, et al. (2005) Antimicrobial resistance in developing countries. Part I: recent trends and current status. Lancet Infect Dis 5:481-93.

7. Okeke IN, Klugman KP, Bhutta ZA, Duse AG, Jenkins $P$, O'Brien TF et al. (2005) Antimicrobial resistance in developing countries. Part II: strategies for containment. Lancet Infect Dis 5:568-80.

8. Morshed MG, Khan NZ, Khan WA, Akbar MS (1986) Multiple drug resistant $S$. typhi in Bangladesh. J Diarrhoeal Dis Res 4:241.

9. Smego RA, Zaidi AKM, Mohammad Z, Bhutta ZA, Hafeez S. (1988) Multiply-resistant Salmonella and Shigella isolates. APMIS Suppl 3:65-7.

10. Chau TT, Campbell JI, Galindo CM, Van Minh Hoang N, Diep TS, Nga TT et al. (2007) Antimicrobial drug resistance of Salmonella enterica Serovar Typhi in Asia and molecular mechanism of reduced susceptibility to the fluoruquinolonees. Antimicrobial and agents and chemotherapy 51(12); 4315-4323.

11. Threfall EJ, Fisher IS, Berghold C, Gerner-Smidt P, Tschepe H, Cormican M et al. (2003) Trends in antimicrobial drug resistance in Salmonella enterica serotypes Typhi and Paratyphi A isolated in Europe, 1999-2001. Int J Antmicrob Agents 22(5):487-91.

12. Hirose K, Tamura K, Sagara H, Watnabe H (2001) Antibiotic susceptibilities of Salmonella enterica Serovar Typhi and S. enterica Serovar Paratyphi A isolated from Patients in Japan. Antmicrob Agents and chemotherapy. 45(3):956-958.

13. Kelesidis T, Kelesidis I, Rafailidis PI, Falagas ME (2007) Counterfeit or substandard antimicrobial drugs: a review of the scientific evidence. J Antimicrob Chemother 60(2):214-36.

14. Wain, JN. Hoa T, Chinh NT, Vinh H, Everett MJ, Diep TS, et al. (1997) Quinolone- resistant Salmonella typhi in Viet Nam: molecular basis of resistance and clinical response to treatment. Clin Infect Dis 25:1404-1410.

15. Threlfall EJ, Ward LR, Skinner JA, Smith HR, Lacey S (1999) Ciprofloxacin-resistant Salmonella typhi and treatment failure. Lancet 353:1590-1591.

16. Parry CM, Ho VA, Phuong le T, Bay PV, Lanh MN, Tung le $\mathrm{T}$ et al. Randomized controlled comparison of ofloxacin, azithromycin, and an ofloxacin-azithromycin combination for treatment of multidrug-resistant and nalidixic acid-resistant typhoid fever. Antimicrob. Agents Chemother. 51:819-825.

17. Chinh, NT, Parry CM, Ly NT, Ha HD, Thong MX, Diep TS, et al. (2000) A randomized controlled comparison of azithromycin and ofloxacin for treatment of multidrugresistant or nalidixic acid-resistant enteric fever. Antimicrob Agents Chemother 44:1855-1859.

18. Griggs DJ, Gensberg K, Piddock LJ (1996) Mutations in gyrA gene of quinolone-resistant Salmonella serotypes isolated from humans and animals. Antimicrob. Agents Chemother. 40:1009-1013.

19. Ling J. Chan MEW, Lam AW, Cheng AF (2003) Mutations in topoisomerase genes of fluoroquinolone-resistant salmonellae in Hong Kong. Antimicrob Agents Chemother 47:3567-3573.

20. Eaves DJ, Randall L, Gray DT, Buckley A, Woodward M.J, White AP et al. (2004) Prevalence of mutations within the quinolone resistance-determining region of gyrA, gyrB, parC, and parE and association with antibiotic resistance in quinolone-resistant Salmonella enterica. Antimicrob. Agents Chemother. 48:4012-4015.

21. Shirakawa T, Acharya B, Kinoshita S, Kumagai, S, Gotoh A, Kawataba M (2006) Decreased susceptibility to fluoroquinolones and gyraAgene mutationin the salmonella enterica serovar Typhi and Paratyphi A isolated in Khatmandu, Nepal, in 2003. Diagn Microbiol Infect Dis 54299-303.

22. Turner AK, Nair S, Wain JN (2006) The acquisition of full fluoroquinoione in Salmonella Typhi by accumulation or point mutationsin the topoisomerase targets. Antimicrob. Agents Chemother 58(4):733-740.

23. Martinez-Martinez L, Pascual A, Jacoby GA (1998) Quinolone resistance from a transferable plasmid. Lancet 351: 797-9.

24. Hopkins KL, Wootton L, Day MR, Threlfall EJ (2007) Plasmid-mediated quinolone resistance determinant 
qnrS1 found in Salmonella enterica strains isolated in the UK. Journal of Antimicrobial Chemotherapy 59(6):10711075.

25. Siddiqui FJ, Rabbani F, Hasan R, Nizami SQ, Bhutta ZA (2006) Typhoid fever in children: some epidemiological considerations from Karachi, Pakistan. Int $\mathrm{J}$ Infect Dis 10(3):215-22.

26. Butt T, Ahmed RN, Salman M, Kazmi SY (2005) Changing trends in drug resistance among typhoid salmonellae in Rawalpindi, Pakistan. Eastern Mediterranean Health Journal. 11:1038-1044.

27. Clinical and laboratory standard institute. (2006).Performance standards for antimicrobial susceptibility testing; sixteenth informational supplement. 26 (1) Clinical and laboratory standard institute.

28. Frank M A, Camilla W, Kare M, Threlfall EJ (2003) Is it time to change Fluoroquinolone breakpoints for Salmonella spp.? Antimicrob Agents Chemother 47(2):827-9.

29. Sur D, Ali M, Von Seidlein L, Manna B, Deen JL, Acosta C, et al. (2007) Comparisons of predictors for typhoid and paratyphoid fever in Kolkata, India. BMC Public Health 7: 289.

30. Walia M, Gaind R, Paul P, Mehta R, Aggarwal P, Kalaivani M. (2006) Age related clinical and microbiological characteristics of enteric fever in India. Transactions of the Royal Society of Tropical Medicine and Hygiene 100(10): 924-948.

31. Raveendran R, Wattal C, Sharma A, Oberoi JK, Parsad KJ, Datta S (2008) High level ciprofloxacin resistance in Salmonella Enterica isolated from blood. Indian Journal of Medical Microbiology 26:50-3.

32. Zhang Q, Sahin O, McDermott PF, Payot S (2006) Fitness of antimicrobial-resistant Campylobacter and Salmonella. Microbes and Infection 8(7): 1972-1978.

33. Holt KE, Thomson NR, Wain JN, Minh DP, Nair S, Hasan $\mathrm{R}$ et al. (2007) Multidrug-resistant Salmonella enterica serovar Paratyphi A harbors IncHI1 plasmids similar to those found in serovar Typhi. J Bacteriology, 189(11): 4257-4264.

34. Mandal S, Mandal M, Pal N (2006) Antibiotic resistance of Salmonella enterica serovar Paratyphi A in India: Emerging and reemerging problem. J. Postgraduate Medicine 52(3): 163-166.

35. Sur D, Von Seidlein L, Manna B, Dutta S, Deb AK, Sarkar BL, Kanungo S et al. (2006) The malaria and typhoid fever burden in the slums of Kolkata, India: data from a prospective community-based study. Trans R. Soc Trop Med Hyg 100(8): 725-733.

36. Luxemburger C, Chau MC, Mai NL, Wain J, Tran TH, Simpson JA, et al. (2001) Risk factors for typhoid fever in the Mekong delta, southern Viet Nam: A case-control study. Trans R Soc Trop Med Hyg 95(1): 19-23.

37. Luby SP, Faizan MK, Fisher-Hoch SP, Syed A, Mintz ED, Bhutta ZA, McCormick JB (1998) Risk factors for typhoid fever in an endemic setting, Karachi, Pakistan. Epidemiol Infect 120(2): 129-138.

38. Srikantiah P, Vafokulov S, Luby SP, Ishmail T, Earhart K, Jennings $G$ et al. (2007) Epidemiology and risk factors for endemic typhoid fever in Uzbekistan. Trop Med Int Health 12(7): 838-847.
Corresponding Author: Rumina Hasan, Department of Pathology \& Microbiology, Aga Khan University, Stadium Road, P.O. Box 3500, Karachi 74800, Pakistan Tele: 92214930051 Ext 4530. Direct line; 9221 4864531, Fax: 9221 4934294, 92 214932095, Email address: rumina.hasan@aku.edu

Conflict of interest: No conflict of interest is declared. 